\title{
Galectin-7 promotes the invasiveness of human oral squamous cell carcinoma cells via activation of ERK and JNK signaling
}

\author{
JIA-PING GUO ${ }^{1}$ and XIAO-GUANG LI $^{2}$ \\ ${ }^{1}$ Department of Stomatology, Wuhan General Hospital of Guangzhou Military Command, \\ Wuhan, Hubei 430070; ${ }^{2}$ Department of Maxillofacial-Head and Neck Oncology, \\ Shanghai Ninth People's Hospital, Shanghai Jiao Tong University School of Medicine, Shanghai 201900, P.R. China
}

Received January 27, 2016; Accepted October 28, 2016

DOI: $10.3892 / 01.2017 .5649$

\begin{abstract}
Galectin-7 is a member of the $\beta$-galactoside-binding protein family, and is highly expressed in oral squamous cell carcinoma (OSCC). The aim of the present study was to investigate the effects of manipulating galectin-7 expression on the biological phenotype of human OSCC cells and the associated molecular mechanisms. Knockdown of endogenous galectin-7 via small interfering RNA (siRNA) was performed and cell proliferation, apoptosis, migration, and invasion were subsequently assessed. The data indicated that galectin-7 silencing had no impact on the proliferation or apoptosis of OSCC cells. However, compared with non-transfected cells, percentage wound closure was significantly lower in galectin-7-silenced cells following $24 \mathrm{~h}$ incubation, indicating decreased cell migration. Furthermore, Matrigel invasion assays demonstrated that galectin-7 knockdown significantly reduced the number of invaded cells, compared with the number in non-transfected cells. Western blot analysis indicated that galectin-7 overexpression resulted in a significant increase in the expression of the proteins matrix metalloproteinase (MMP)-2 and MMP-9. The invasive abilities of cells overexpressing galectin-7 significantly decreased following co-transfection with MMP-2- or MMP-9-specific siRNA. Increasing galectin-7 expression significantly enhanced the phosphorylation of extracellular signal-related kinase (ERK) $1 / 2$ and c-Jun N-terminal kinase (JNK) 1/2. Pharmacological inhibition of ERK or JNK activity significantly suppressed the invasiveness of galectin-7-overexpressing cells and abrogated the upregulation of MMP-2 and MMP-9. Taken together, the results of the current study provide novel evidence for the pro-invasive activity of galectin-7 in OSCC cells, which is
\end{abstract}

Correspondence to: Dr Xiao-Guang Li, Department of Maxillofacial-Head and Neck Oncology, Shanghai Ninth People's Hospital, Shanghai Jiao Tong University School of Medicine, 280 Mohe Road, Shanghai 201900, P.R China

E-mail: dtqetiqwpoti@qq.com

Key words: cell proliferation, tumor invasion, oral squamous cell carcinoma, signaling pathways associated with activation of ERK and JNK signaling and the induction of MMP-2 and MMP-9.

\section{Introduction}

Oral squamous cell carcinoma (OSCC) is one of the most common malignancies in the world, and has a high mortality rate (1). Invasive growth via the lymphatic route is a typical feature of OSCC (2) and lymph node involvement status has been identified as a reliable prognostic indicator in OSCC patients $(3,4)$. Therefore, a number of studies are underway in order to investigate the molecular mechanisms involved in regulating OSCC invasiveness (5).

Galectin-7 is a member of the $\beta$-galactoside-binding protein family. It is predominantly expressed in epithelial cells within healthy tissue and plays an important role in epithelial development and homeostasis $(6,7)$. Galectin-7 expression may be altered in epithelial cancer, therefore it may serve an important role in cancer progression (8). The exact role of galectin-7 may vary in different types of cancers; it may play distinct and even opposing roles in tumor development. For example, in human gastric cancer specimens, galectin-7 is underexpressed due to epigenetic modifications and this suppresses the proliferation and invasion of gastric cancer cells (9). By contrast, in high-grade breast cancer galectin-7 is overexpressed, facilitating the spontaneous metastasis of breast cancer cells in preclinical mouse models (10). The tumor-promoting role of galectin-7 has also been noted in ovarian cancer cells (11) and cervical cancer cells (12).

Previous studies have demonstrated that galectin-7 increases the expression of matrix metalloproteinases (MMPs), especially MMP-9, thus modulating the invasiveness of cancer cells $(11,12)$. Additionally, OSCC tissues exhibit increased MMP-2 and MMP-9 activity compared with adjacent healthy tissues (13). It has previously been demonstrated that MMPs serve a critical role in the invasion and metastasis of oral cancer (14). Alves et al (15) reported that galectin-7 is highly expressed in OSCC and its expression is significantly correlated with the histological grade of disease. These findings suggest that galectin-7 may contribute to OSCC invasiveness by modulating the expression of MMP-2 and MMP-9. The present study investigated the effects of manipulating galectin-7 expression on the biological phenotypes of human 
OSCC cells and evaluated the involvement of MMP-2 and MMP-9 on the action of galectin-7.

\section{Materials and methods}

Cell culture and treatment. The human OSCC cell lines SCC- 4 and SCC-9 were purchased from the American Type Culture Collection (ATCC; Manassas, VA, USA). All cells were maintained at $37^{\circ} \mathrm{C}$ in $5 \% \mathrm{CO}_{2}$ in Dulbecco's Modified Eagle's Medium supplemented with $10 \%$ fetal bovine serum (FBS), $1 \mathrm{mmol} / \mathrm{l} \mathrm{L}$-glutamine, and $100 \mathrm{U} / \mathrm{ml}$ penicillin, $100 \mu \mathrm{g} / \mathrm{ml}$ streptomycin (all from Invitrogen; Thermo Fisher Scientific, Inc., Waltham, MA, USA). For inhibitor experiments, cells were pretreated with the c-Jun N-terminal kinase (JNK) inhibitor SP600125 (10 $\mu \mathrm{M}$; Calbiochem; EMD Millipore, Billerica, MA, USA), extracellular signal-related kinase (ERK) inhibitor PD98059 (10 $\mu \mathrm{M}$; Calbiochem; EMD Millipore), or $0.1 \%$ dimethyl sulfoxide (DMSO) used as vehicle control $1 \mathrm{~h}$ before transfection of galectin-7-expressing plasmid.

Plasmids, small interfering RNA (siRNA), and transfection. A galectin-7-expressing plasmid (pCEP4-GAL7) was purchased from Addgene (Cambridge, MA, USA) and an empty vector (pCEP4) was also purchased (Invitrogen; Thermo Fisher Scientific, Inc.) Galectin-7 siRNA, MMP-2 siRNA, MMP-9 siRNA, and negative control siRNA were obtained from Santa Cruz Biotechnology (Dallas, TX, USA). For overexpression or knockdown of galectin-7, cells were seeded onto 6-well plates $\left(4 \times 10^{5}\right.$ cells/well) and transfected with $1 \mu \mathrm{g}$ pCEP4-GAL7, $1 \mu \mathrm{g} \mathrm{pCEP} 4$ and $50 \mathrm{nM}$ galectin-7 siRNA, or $50 \mathrm{nM}$ control siRNA using Lipofectamine ${ }^{\circledR} 2000$ (Invitrogen; Thermo Fisher Scientific, Inc.), according to the manufacturer's instructions. Cells were incubated for $24 \mathrm{~h}$, and subsequently collected for further experiments. To validate the involvement of MMP-2 and MMP-9, cells were co-transfected with $1 \mu \mathrm{g}$ pCEP4-GAL7 and $50 \mathrm{nM}$ MMP-2 siRNA, MMP-9 siRNA, or control siRNA, and tested for invasive ability following incubation for $24 \mathrm{~h}$.

Cell proliferation assay. Cell proliferation was measured using the MTT assay. Transfected cells were detached and re-seeded onto 96 -well plates ( $2 \times 10^{3} /$ well). Following incubation for 1, 3, and 5 days, $0.5 \mathrm{mg} / \mathrm{ml} \mathrm{MTT} \mathrm{(Sigma-Aldrich;} \mathrm{Merck} \mathrm{Millipore,}$ Darmstadt, Germany) was added to the culture and incubated for additional $4 \mathrm{~h}$ at $37^{\circ} \mathrm{C}$. Formazan crystals were dissolved in DMSO. Absorbance was measured at $570 \mathrm{~nm}$ using a multi-plate reader.

Apoptosis detection assay. Apoptosis analysis was performed using the Annexin V-FITC Apoptosis Detection kit (Nanjing KeyGen Biotech Co., Nanjing, China), according to the manufacturer's instructions. In brief, cells were incubated with a staining solution containing fluorescein isothiocyanate (FITC)-conjugated annexin-V and propidium iodide (PI) for $10 \mathrm{~min}$ at $4^{\circ} \mathrm{C}$ in the dark. The percentage of apoptotic cells was determined using a FACScan flow cytometer with the CellQuest software (BD Biosciences, San Jose, CA, USA).

Wound-healing assay. Cells were seeded onto 6-well plates and allowed to grow to $~ 95 \%$ confluence. A wound was made in the monolayer using a $100-\mu \mathrm{l}$ pipette tip. The culture was washed to remove cellular debris and incubated for $24 \mathrm{~h}$ at $37^{\circ} \mathrm{C}$. Cells were imaged using a phase contrast microscope at different time points. The extent of wound closure was quantified by measuring its area before migration, and $24 \mathrm{~h}$ after migration. Results were expressed as percentage of wound closure.

Transwell invasion assay. Invasion assays were performed using Transwell chambers, which were coated with Matrigel (BD Biosciences) $24 \mathrm{~h}$ prior to use. The cells were subsequently harvested and resuspended in serum-free medium containing $1 \%$ bovine serum albumin (Sigma-Aldrich; Merck Millipore). The cell suspension was added to the upper chamber and the lower chamber was filled with culture medium containing $10 \% \mathrm{FBS}$. After incubation for $24 \mathrm{~h}$ at $37^{\circ} \mathrm{C}$, cells on the upper surface of the chamber were removed using a cotton swab. Invaded cells on the lower surface were fixed in $4 \%$ formaldehyde, stained with $0.5 \%$ crystal violet, and counted under a microscope.

Reverse transcription-quantitative polymerase chain reaction (RT- $q P C R)$ analysis. Total RNA was extracted from cells using the TRIzol reagent following the manufacturer's instructions (Invitrogen; Thermo Fisher Scientific, Inc.). Reverse transcription was performed using the PrimeScript First Strand cDNA Synthesis kit (Takara Biotechnology Co., Dalian, China). RT-qPCR was performed on an ABI 7500 Fast Real-Time PCR System (Applied Biosystems; Thermo Fisher Scientific, Inc.) with SYBR-Green detection mix (Takara Biotechnology Co.). The following primers were used in the current study: Galectin-7 forward 5'-TTGCTCCTTGCTGTT GAAGACCAC-3', and reverse 5'-AGGTTCCATGTAAAC CTGCTGTGC-3' (16); glyceraldehyde-3-phosphate dehydrogenase (GAPDH) forward, 5'-TGACTTCAACAGCGACAC CCA-3'; and reverse, 5'-CACCCTGTTGCTGTAGCCAAA-3'. PCR conditions were as follows: $95^{\circ} \mathrm{C}$ for $5 \mathrm{~min}$, followed by 40 cycles of $95^{\circ} \mathrm{C}$ for $10 \mathrm{sec}, 64^{\circ} \mathrm{C}$ for $30 \mathrm{sec}$, and $72^{\circ} \mathrm{C}$ for $30 \mathrm{sec}$. The relative galectin-7 mRNA level was calculated using the $2^{-\Delta \Delta C q}$ method (17) following normalization against the level of GAPDH.

Western blot analysis. Cells were lysed in radioimmunoprecipitation assay buffer (phosphate buffer solution, 1\% NP40, $0.5 \%$ sodium deoxycholate, $0.1 \%$ sodium dodecyl sulphate) containing a protease inhibitor cocktail (Cell Signaling Technology, Inc., Danvers, MA, USA) on ice for $30 \mathrm{~min}$. After centrifugation at $15,000 \mathrm{x} \mathrm{g}$ for $20 \mathrm{~min}$, the supernatant was collected and protein concentrations were measured using a protein assay kit (Bio-Rad Laboratories, Inc., Hercules, CA, USA). Cell lysates were separated by sodium dodecyl sulfate-polyacrylamide gel electrophoresis and transferred onto nitrocellulose membranes. Membranes were probed with the following antibodies at 1:300 dilution: Rabbit anti-galectin-7 monoclonal antibody (cat. no. ab108623), rabbit anti-MMP-2 polyclonal antibody (cat. no. ab97779), mouse anti-MMP-9 monoclonal antibody (cat. no. ab119906), rabbit anti-GAPDH monoclonal antibody (cat. no. ab181602; all from Abcam, Cambridge, MA, USA), rabbit anti-phospho-ERK1/2 polyclonal antibody (cat. no. 9101), rabbit anti-ERK1/2 polyclonal antibody (cat. no. 9102), rabbit anti-phospho-JNK monoclonal 
antibody (cat. no. 4668), rabbit anti-JNK polyclonal antibody (cat. no. 9252), rabbit anti-phospho-p38 monoclonal antibody (cat. no. 4511) and rabbit anti-p38 monoclonal antibody (cat. no. 8690; all from Cell Signaling Technology, Inc.). Horseradish peroxidase-conjugated secondary antibodies (cat. nos. sc-2004 and sc-2005; Santa Cruz Biotechnology, Inc.) were diluted at 1:2,000 prior to use. Proteins were visualized using an enhanced chemiluminescence kit (Pierce Biotechnology, Inc., Rockford, IL, USA). The blots were quantified by densitometry with the Quantity One software (Bio-Rad Laboratories).

Statistical analysis. Data are expressed as mean \pm standard deviation. Statistical differences were examined using one-way analysis of variance (ANOVA) followed by Tukey's post hoc test. $\mathrm{P}<0.05$ was considered to indicate a statistically significant difference.

\section{Results}

Galectin-7 silencing has no impact on cell proliferation or apoptosis in OSCC cells. To analyze the role of galectin-7 in the proliferation of OSCC cells, galectin-7-specific siRNA was transiently transfected into SCC-4 and SCC-9 cell lines. The delivery of galectin-7 siRNA significantly decreased mRNA and protein levels of endogenous galectin-7 in both SCC-4 and SCC-9 cells (Fig. 1A and B; P<0.05). The results of the MTT assay demonstrated that this downregulation of galectin-7 did not significantly affect SCC-4 and SCC-9 cell proliferation compared with non-transfected cells over a 5-day period (Fig. 1C). Annexin-V/PI staining analysis identified comparable percentages of apoptotic cells in non-transfected and galectin-7 siRNA-transfected cells (Fig. 1D).

Galectin-7 knockdown attenuates the migration and invasion of OSCC cells. The effect of galectin-7 downregulation on the invasive properties of OSCC cells was then analyzed. Galectin-7 silencing caused a significant decline in cell motility during in vitro wound-healing assays. Compared to non-transfected SCC-4 cells, the percentage wound closure was significantly lower in galectin-7-silenced SCC-4 cells $24 \mathrm{~h}$ following incubation $(18.5 \pm 3.2 \%$ vs. $54.4 \pm 6.4 \%, \mathrm{P}<0.05$; Fig. 2A). Similarly, galectin-7 siRNA transfection resulted in a significant reduction in the motility of SCC-9 cells $(\mathrm{P}<0.05)$. Matrigel invasion assays demonstrated that galectin-7 knockdown significantly reduced the numbers of invaded cells by $>60 \%$, compared with non-transfected cells ( $\mathrm{P}<0.05$; Fig. 2B).

Overexpression of galectin-7 accelerates the migration and invasion of OSCC cells. Further tests confirmed the effect of increased galectin-7 on the migration and invasion of OSCC cells. Transfection of the plasmid pCEP4-GAL7 into SCC-4 and SCC-9 cells led to a significant increase in galectin-7 expression compared with non-transfected cells (Fig. 3A). This increase in galectin-7 expression in turn significantly increased OSCC cell migration and invasion $(\mathrm{P}<0.05$; Figs. 3B and $\mathrm{C})$.

Upregulation of MMP-2 and MMP-9 mediates the pro-invasive activity of galectin-7. A possible association between galectin-7-mediated invasiveness and MMP-2 and MMP-9

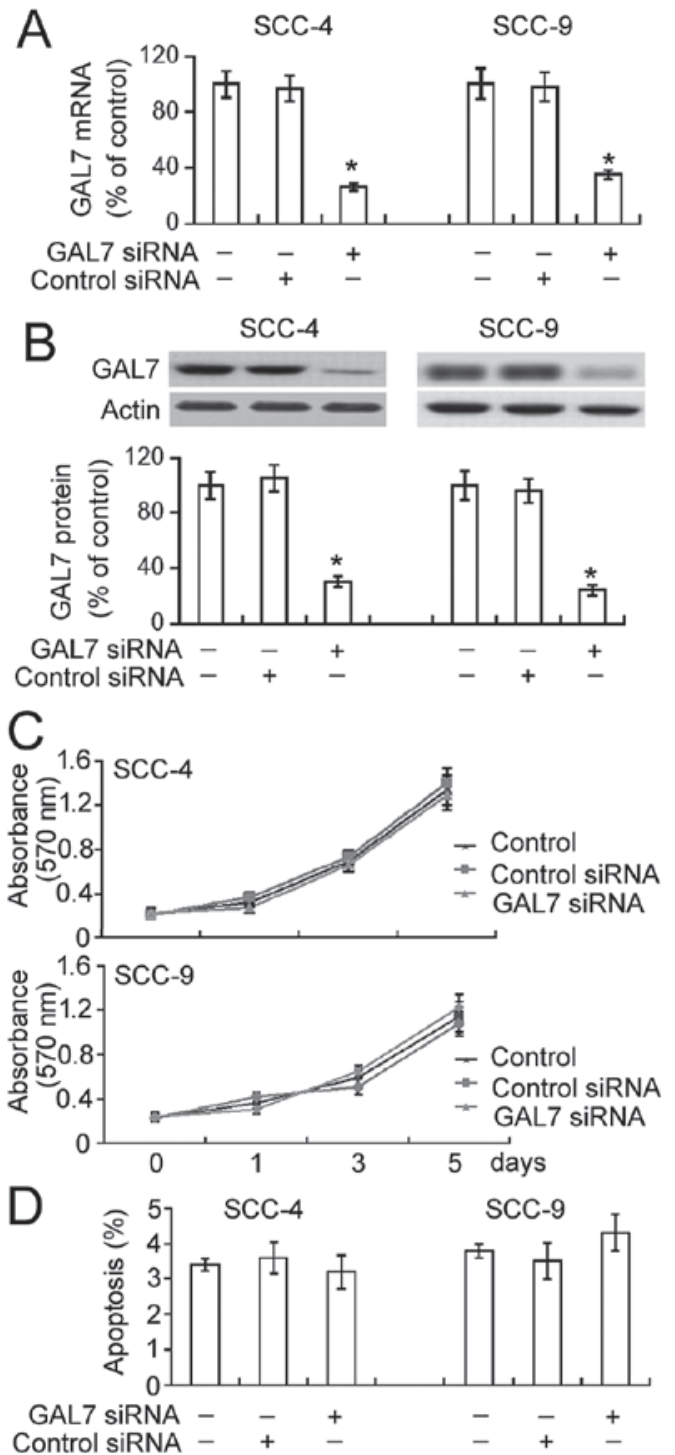

Figure 1. GAL7 silencing does not affect the proliferation and apoptosis of OSCC cells. (A) RT-qPCR and (B) western blot analysis of galectin-7 mRNA and protein levels, respectively, in SCC-4 and SCC-9 cells transfected with control or galectin-7 siRNA. Bar graphs represent mean \pm SD of three independent experiments. ${ }^{*} \mathrm{P}<0.05$ vs. non-transfected cells. (C) The viability and (D) apoptosis of SCC-4 and SCC-9 cells transfected with control or galectin-7 siRNA were determined by MTT assay and annexin-V/PI staining analysis, respectively. GAL7, galectin-7; OSCC, oral squamous cell carcinoma; RT-qPCR, reverse transcription-quantitative polymerase chain reaction; PI, propidium iodide; siRNA, small interfering RNA.

upregulation was investigated. Western blot analysis demonstrated that galectin-7 overexpression resulted in a 3-5-fold increase in MMP-2 protein and 2-3-fold increase in MMP-9 protein expression in both SCC-4 and SCC-9 cells (Fig. 4A). Transwell invasion assay demonstrated that the invasiveness of SCC-4 and SCC-9 cells overexpressing galectin-7 was significantly decreased by co-transfection with MMP-2 or MMP-9-specific siRNA (P<0.05; Fig. 4B).

Galectin-7 promotes OSCC cell invasion via activation of $E R K$ and JNK signaling. Finally, the signaling pathways involved in the action of galectin-7 were investigated. As shown in Fig. 5A, increasing galectin-7 expression markedly enhanced the phosphorylation of ERK1/2 and JNK1/2 


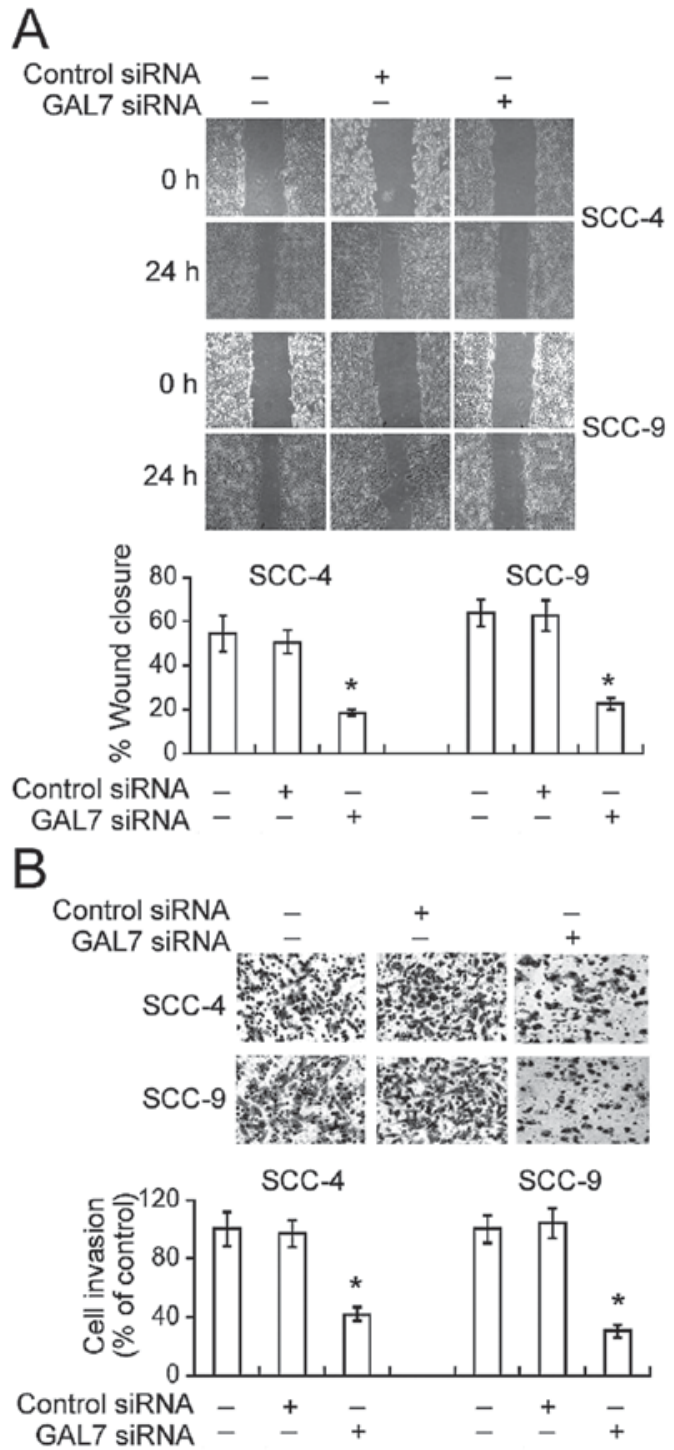

Figure 2. GAL7 knockdown attenuates OSCC cell migration and invasion. (A) Cells transfected with control or GAL7 siRNA were subjected to a wound-healing assay. Top panels: Representative images of cells were taken before and $24 \mathrm{~h}$ after scratching. Bottom panels: Bar graphs show the mean percentage of three independent experiments. (B) Transwell invasion assay. Cells transfected with control or GAL7 siRNA were tested for invasiveness in Matrigel-coated transwells. Top panels: Representative images of invasion assay are shown. Bottom panels: Bar graphs represent quantitative data from three independent experiments. ${ }^{~} \mathrm{P}<0.05$ vs. non-transfected cells. GAL7, galectin-7; OSCC, oral squamous cell carcinoma; siRNA, small interfering RNA.

in SCC-4 and SCC-9 cells, without altering total levels of ERK1/2 and JNK1/2. No change in p38 phosphorylation levels was detected. Notably, the pharmacological inhibition of ERK or JNK activity significantly suppressed the invasiveness of galectin-7-overexpressing SCC-4 and SCC-9 cells $(\mathrm{P}<0.05$; Fig. 5B) and abrogated the upregulation of MMP-2 and MMP-9 (P<0.05; Fig. 5C).

\section{Discussion}

Matsukawa et al (18) reported previously that adenoviral delivery of the galectin-7 gene may induce modest apoptosis and reduce the viability of human OSCC HSC3 cells.

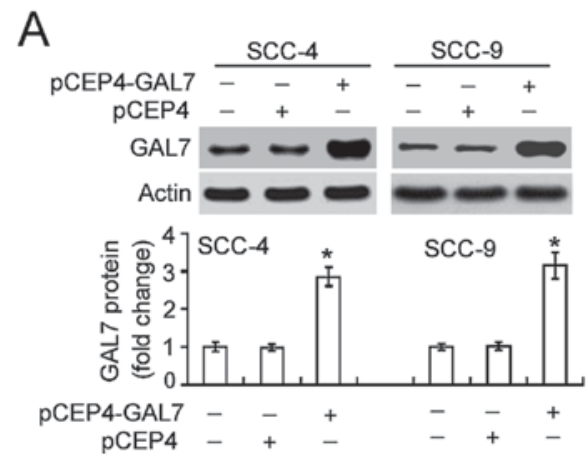

B

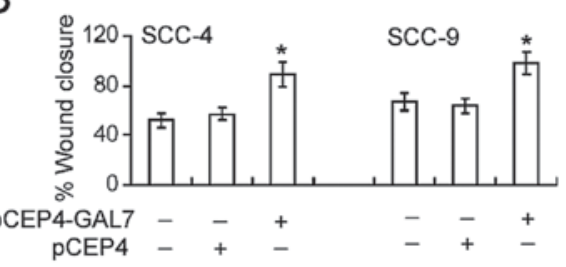

$\mathrm{C}$

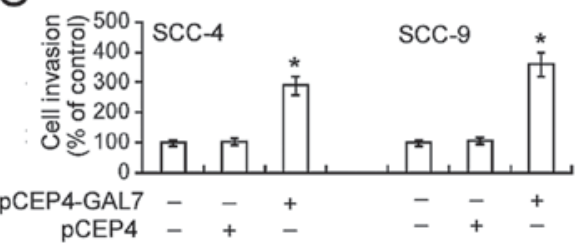

Figure 3. Overexpressing GAL7 accelerates the migration and invasion of OSCC cells. (A) Western blot analysis of galectin-7 in OSCC cells transfected with vector (pCEP4) or GAL7-expressing plasmid (pCEP4-GAL7). Bar graphs represent quantitative data from three independent experiments. (B) The migration and (C) invasion capacities of vector-or GAL7-expressing cells were determined by wound-healing assay and Transwell invasion assay, respectively. Bar graphs represent quantitative data from three independent experiments. ${ }^{*} \mathrm{P}<0.05$ vs. non-transfected cells. GAL7-galectin-7; OSCC, oral squamous cell carcinoma.

However, knockdown of galectin-7 using antisense galectin-7 oligonucleotides demonstrated no significant effects on cell viability. In the current study, the biological roles of galectin-7 in two other OSCC cell lines were explored, and targeted reduction of galectin-7 via siRNA technology did not alter viability and spontaneous apoptosis in SCC-4 and SCC-9 cells. These results suggest that galectin-7 is not required for the maintenance of OSCC cell viability. The anti-viability effect elicited by overexpression of galectin-7 may only reflect a non-specific cytotoxicity, as the potential cytotoxic activity of galectin-7 overexpression on healthy human cells was not tested in the current study.

The ability of galectin-7 to modulate cell behavior seems to be cell-dependent. Previous studies have demonstrated that overexpressing galectin-7 inhibits the proliferation of several specific cancer cells such as gastric cancer cells (9) and colon carcinoma cells (19). However, in other cancer cells including epithelial ovarian cancer (20), galectin-7 was involved in cell proliferation, as its downregulation inhibited the proliferation of A2780-PAR ovarian cancer cell.

Metastasis is the main cause of cancer-associated mortality. Galectin-7 exhibits the ability to modulate the metastatic phenotype of several types of cancer cells $(10,12,21)$. Demers et al (21) demonstrated that ectopic expression of 


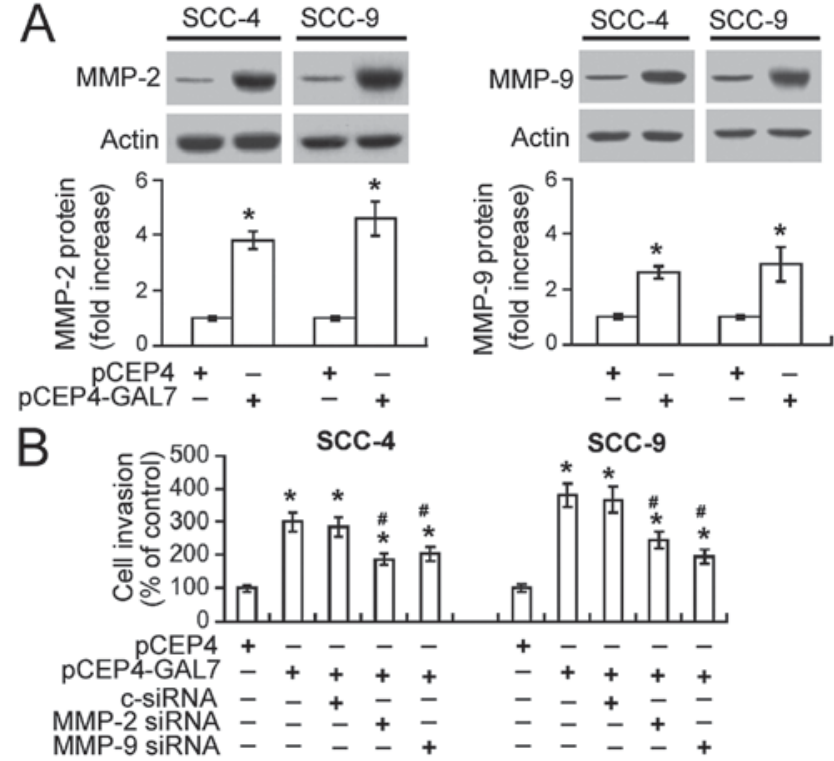

Figure 4. Upregulation of MMP-2 and MMP-9 mediates the pro-invasive activity of GAL7. (A) Western blot analysis of MMP-2 and MMP-9 protein expression in cells transfected with vector or GAL7-expressing plasmid. Bar graphs represent quantitative data from three independent experiments. "P $<0.05$ vs. vector-transfected cells. (B) The invasiveness of cells transfected with indicated constructs was determined by Transwell invasion assay. ${ }^{*} \mathrm{P}<0.05$ vs. vector-transfected cells; ${ }^{*} \mathrm{P}<0.05$ vs. cells transfected with GAL7-expressing plasmid alone. GAL7, galectin-7; MMP, matrix metalloproteinase.

galectin-7 increases the invasiveness of lymphoma, accelerates the development of thymic lymphoma, and previously identified that overexpressing galectin-7 enhances the metastatic growth of breast cancer cells in the lungs and bones in two different mouse models (10). Enforced expression of galectin-7 also promotes the invasiveness of human HeLa cervical epithelial adenocarcinoma cells (12). The results of the present study are consistent with results from previous studies, as they demonstrated that galectin-7 has the ability to modulate the invasive properties of OSCC cells. Knockdown of galectin-7 suppressed the migration and invasion of SCC-4 and SCC-9 cells, whereas overexpressing galectin-7 increased them. Taken together, these findings indicate that galectin-7 is a potential target for the treatment of tumor dissemination in OSCC.

Compelling evidence suggests that the induction of MMPs plays a pivotal role in the OSCC invasiveness. For instance, Bedal et al (22) have previously reported that collagen XVI facilitates the invasion of OSCC cells by inducing MMP-9 expression. It has previously been suggested that the downregulation of MMP-2 and MMP-9 may account for the decreased invasiveness of OSCC cells due to the knockdown of BubR1, a critical component of spindle assembly checkpoint (23). Inhibiting MMP-2 and MMP-9 expression has also been demonstrated to mediate the anti-invasive effects of curcumin (a natural polyphenolic compound) in OSCC cells (24). In line with its pro-invasive activity, galectin-7 expression increases the expression of MMP-9 in several cancer cells $(12,16,21)$. The current study investigated the effects of MMP-2 and MMP-9 on galectin-7 action in OSCC cells. Galectin-7 overexpression resulted in the significant upregulation of MMP-2 and MMP-9.

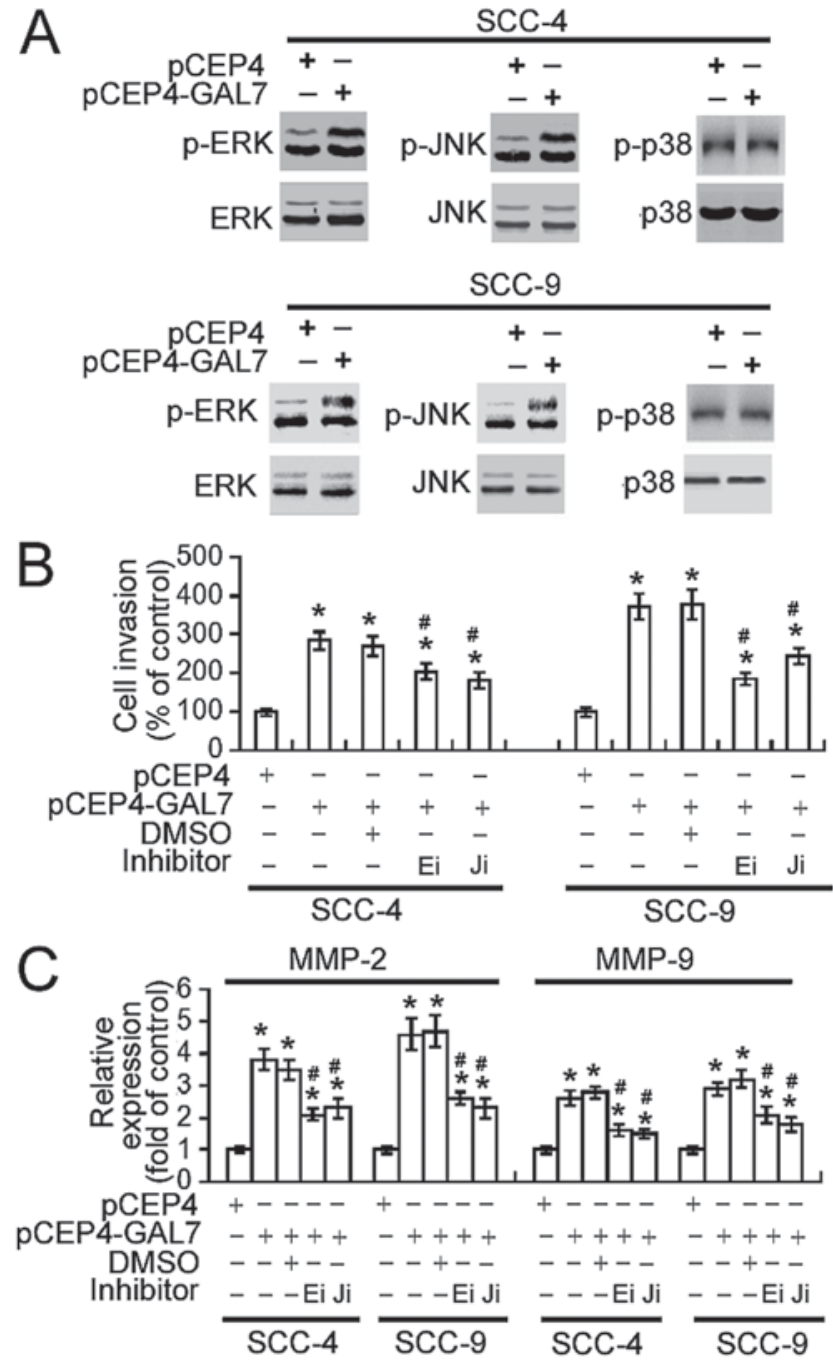

Figure 5. GAL7 promotes OSCC cell invasion via activation of ERK and JNK signaling. (A) Western blot analysis of signaling proteins in cells transfected with vector or GAL7-expressing plasmid. Representative blots from three independent experiments are shown. (B and C) Cells were transfected with vector or GAL7-expressing plasmid with or without prior treatment with an ERK inhibitor (Ei) or JNK inhibitor (Ji). (B) Cell invasiveness and (C) MMP-2 and MMP-9 expression were determined by Transwell invasion assay and Western blot analysis, respectively. Bar graphs represent quantitative data from three independent experiments. ${ }^{*} \mathrm{P}<0.05$ vs. vector-transfected cells; ${ }^{\#} \mathrm{P}<0.05$ vs. cells transfected with GAL7-expressing plasmid alone. GAL7, galectin 7; OSCC, oral squamous cell carcinoma; MMP, matrix metalloproteinase; ERK, extracellular signal-related kinase; JNK, c-jun N-terminal kinase.

Most importantly, silencing MMP-2 or MMP-9 significantly impaired the invasiveness of OSCC cells that overexpressed galectin-7. Thus MMP-2 and MMP-9 may be required for the galectin-7-mediated invasiveness of OSCC cells.

To gain a better insight into the function of galectin-7 in OSCC cell invasiveness, the signaling pathways involved were analyzed. Since mitogen-activated protein kinase (MAPK) pathways are implicated in the invasion of oral cancer cells $(25,26)$ and galectin- 7 may activate p38 MAPK signaling in cervical cancer cells (12), the current study investigated the importance of MAPK signaling in mediating galectin-7 action. The results of the present study demonstrated that galectin-7 overexpression leads to the phosphorylation and activation of ERKs and JNKs, but not p38 MAPK, in SCC-4 
and SCC-9 cells. Interestingly, the pharmacological inhibition of ERK or JNK activity significantly attenuated OSCC cell invasiveness induced by galectin-7 overexpression. Moreover, galectin-7-mediated upregulation of MMP-2 and MMP-9 was compromised by pretreatment with the ERK or JNK inhibitors. Taken together, these results suggest that galectin-7 promotes the invasiveness of OSCC cells largely by inducing the expression of MMP-2 and MMP-9 via activation of ERK and JNK signaling.

In conclusion, the current study provides novel evidence demonstrating the pro-invasive activity of galectin-7, which is associated with increased MMP-2 and MMP-9 expression, in OSCC cells. Furthur studies are required to investigate the utility of galectin-7 as a target for the treatment of metastatic OSCC.

\section{References}

1. Torre LA, Bray F, Siegel RL, Ferlay J, Lortet-Tieulent J and Jemal A: Global cancer statistics, 2012. CA Cancer J Clin 65: 87-108, 2015.

2. Ziober AF, Falls EM and Ziober BL: The extracellular matrix in oral squamous cell carcinoma: Friend or foe? Head Neck 28: 740-749, 2006

3. Künzel J, Mantsopoulos K, Psychogios G, Grundtner P, Koch M and Iro H: Lymph node ratio as a valuable additional predictor of outcome in selected patients with oral cavity cancer. Oral Surg Oral Med Oral Pathol Oral Radiol 117: 677-684, 2014.

4. Kim SY, Nam SY, Choi SH, Cho KJ and Roh JL: Prognostic value of lymph node density in node-positive patients with ora squamous cell carcinoma. Ann Surg Oncol 18: 2310-2317, 2011.

5. Sasahira T, Kirita T and Kuniyasu H: Update of molecular pathobiology in oral cancer: A review. Int J Clin Oncol 19: 431-436, 2014.

6. Magnaldo T, Fowlis D and Darmon M: Galectin-7, a marker of all types of stratified epithelia. Differentiation 63: 159-168, 1998.

7. Rondanino C, Poland PA, Kinlough CL, Li H, Rbaibi Y, Myerburg MM, Al-bataineh MM, Kashlan OB, Pastor-Soler NM, Hallows KR, et al: Galectin-7 modulates the length of the primary cilia and wound repair in polarized kidney epithelial cells. Am J Physiol Renal Physiol 301: F622-F633, 2011.

8. St-Pierre Y, Campion CG and Grosset AA: A distinctive role for galectin-7 in cancer? Front Biosci (Landmark Ed) 17: 438-450, 2012.

9. Kim SJ, Hwang JA, Ro JY, Lee YS and Chun KH: Galectin-7 is epigenetically-regulated tumor suppressor in gastric cancer. Oncotarget 4: 1461-1471, 2013.

10. Demers M, Rose AA, Grosset AA, Biron-Pain K, Gaboury L, Siegel PM and St-Pierre Y: Overexpression of galectin-7, a myoepithelial cell marker, enhances spontaneous metastasis of breast cancer cells. Am J Pathol 176: 3023-3031, 2010.

11. Labrie M, Vladoiu MC, Grosset AA, Gaboury L and St-Pierre Y: Expression and functions of galectin-7 in ovarian cancer. Oncotarget 5: 7705-7721, 2014 .
12. Park JE, Chang WY and Cho M: Induction of matrix metalloproteinase- 9 by galectin-7 through p38 MAPK signaling in HeLa human cervical epithelial adenocarcinoma cells. Oncol Rep 22: 1373-1379, 2009

13. Patel BP, Shah PM, Rawal UM, Desai AA, Shah SV, Rawal RM and Patel PS: Activation of MMP-2 and MMP-9 in patients with oral squamous cell carcinoma. J Surg Oncol 90: 81-88, 2005.

14. Thomas GT, Lewis MP and Speight PM: Matrix metalloproteinases and oral cancer. Oral Oncol 35: 227-233, 1999.

15. Alves PM, Godoy GP, Gomes DQ, Medeiros AM, de Souza LB, da Silveira EJ, Vasconcelos MG and Queiroz LM: Significance of galectins-1, -3, -4 and -7 in the progression of squamous cell carcinoma of the tongue. Pathol Res Pract 207: 236-240, 2011.

16. Demers M, Biron-Pain K, Hébert J, Lamarre A, Magnaldo T and St-Pierre Y: Galectin-7 in lymphoma: Elevated expression in human lymphoid malignancies and decreased lymphoma dissemination by antisense strategies in experimental model. Cancer Res 67: 2824-2829, 2007.

17. Giulietti A, Overbergh L, Valckx D, Decallonne B, Bouillon R and Mathieu C: An overview of real-time quantitative PCR: Applications to quantify cytokine gene expression. Methods 25: 386-401, 2001

18. Matsukawa S, Morita K, Negishi A, Harada H, Nakajima Y, Shimamoto H, Tomioka H, Tanaka K, Ono M, Yamada T and Omura K: Galectin-7 as a potential predictive marker of chemoand/or radio-therapy resistance in oral squamous cell carcinoma. Cancer Med 3: 349-361, 2014.

19. Ueda S, Kuwabara I and Liu FT: Suppression of tumor growth by galectin-7 gene transfer. Cancer Res 64: 5672-5676, 2004.

20. Kim HJ, Jeon HK, Lee JK, Sung CO, Do IG, Choi CH, Kim TJ, Kim BG, Bae DS and Lee JW: Clinical significance of galectin-7 in epithelial ovarian cancer. Anticancer Res 33: 1555-1561, 2013.

21. Demers M, Magnaldo T and St-Pierre Y: A novel function for galectin-7: Promoting tumorigenesis by up-regulating MMP-9 gene expression. Cancer Res 65: 5205-5210, 2005.

22. Bedal KB, Grässel S, Oefner PJ, Reinders J, Reichert TE and Bauer R: Collagen XVI induces expression of MMP9 via modulation of AP-1 transcription factors and facilitates invasion of oral squamous cell carcinoma. PLoS One 9: e86777, 2014

23. Chou CK, Wu CY, Chen JY, Ng MC, Wang HM, Chen JH, Yuan SS, Tsai EM, Chang JG and Chiu CC: BubR1 acts as a promoter in cellular motility of human oral squamous cancer cells through regulating MMP-2 and MMP-9. Int J Mol Sci 16: 15104-15117, 2015

24. Lee AY, Fan CC, Chen YA, Cheng CW, Sung YJ, Hsu CP and Kao TY: Curcumin inhibits invasiveness and epithelial-mesenchymal transition in oral squamous cell carcinoma through reducing matrix metalloproteinase 2,9 and modulating p53-E-cadherin pathway. Integr Cancer Ther 14: 484-490, 2015.

25. Liu FY, Safdar J, Li ZN, Fang QG, Zhang X, Xu ZF and Sun CF: CCR7 regulates cell migration and invasion through MAPKs in metastatic squamous cell carcinoma of head and neck. Int J Oncol 45: 2502-2510, 2014.

26. Chen PN, Hsieh YS, Chiang CL, Chiou HL, Yang SF and Chu SC: Silibinin inhibits invasion of oral cancer cells by suppressing the MAPK pathway. J Dent Res 85: 220-225, 2006. 